\title{
Genetic Variability of Pigeonpea (Cajanus cajan (L.) Millsp.) for Water Logging and Salinity Tolerance under in vitro and in vivo Conditions
}

\author{
Sarvjeet Singh ${ }^{1^{*}}$, Pooja Grover ${ }^{1}$, Jagmeet Kaur ${ }^{1}$, Inderjit Singh ${ }^{1}$, Jasdeep Kaur ${ }^{1}$, \\ Pritpal Singh', O. P. Choudhary², Anupama Hingane ${ }^{3}$, C. V. Sameer Kumar ${ }^{3}$ \\ and K. B. Saxena ${ }^{3}$ \\ ${ }^{1}$ Department of Plant Breeding and Genetics, Punjab Agricultural University, Ludhiana, India. \\ ${ }^{2}$ Department of Soil Science, Punjab Agricultural University, Ludhiana, India. \\ ${ }^{3}$ International Crops Research Institute for the Semi-Arid Tropics, Patancheru, India.
}

\section{Authors' contributions}

This work was carried out in collaboration between all authors. Authors PG, IS and OPC conducted field experiments. Authors Jagmeet Kaur and Jasdeep Kaur conducted laboratory experiments. Authors PG and AH prepared the first draft. Authors SS, OPC and KBS designed the experiments. Author PS performed the statistical analysis. Authors SS, CVSK and KBS prepared the final draft. All authors read and approved the final manuscript.

Article Information

DOI: $10.9734 / A J E A / 2016 / 24071$

Editor(s):

(1) Slawomir Borek, Department of Plant Physiology, Adam Mickiewicz University, Poland.

Reviewers:

(1) Ozlem Onal Asci, Ordu University, Turkey. (2) Vincent Ezin, University of Abomey-Calavi, Benin. (3) Badar-uz-Zaman, National Agriculture Research Centre, Islamabad, Pakistan.

(4) Biljana Bojovic, University of Kragujevac, Serbia. Complete Peer review History: http://sciencedomain.org/review-history/14023

Original Research Article

Received $2^{\text {nd }}$ January 2016 Accepted $16^{\text {th }}$ March 2016

Published $5^{\text {th }}$ April 2016

\begin{abstract}
Pigeonpea (Cajanus cajan (L.) Millsp.) genotypes acquired from diverse sources, were screened for water logging and salinity tolerance under laboratory and field conditions. Analysis of variance revealed significant differences among the genotypes for various traits. Based on seedling vigor index, six genotypes ( $\mathrm{AL}$ 1756, $\mathrm{AL}$ 1849, $\mathrm{AH}-06-7, \mathrm{H}-2000-14, \mathrm{H}-2003-14, \mathrm{CCP} 5028$ ) were found highly tolerant, 10 tolerant, 24 moderately tolerant, 12 sensitive and eight highly sensitive to water logging. In case of salinity treatment, seven genotypes ( $\mathrm{AL} 1849, \mathrm{AH}-06-7, \mathrm{H}-2000-14, \mathrm{H}-2001-25$,
\end{abstract}


H-2003-14, ICP 5028, JBP 110B) were found tolerant, 14 moderately tolerant, 17 sensitive and 22 highly sensitive. Mean values for germination percentage, seedling length, seedling dry weight and seedling vigor index were generally lower in salinity treatment than water logging treatment. Based on results of laboratory studies, 28 genotypes were screened in pot/field for tolerance to water logging and salinity and were categorized on the basis of plant survival percentage after treatments. Based on plant survival, four genotypes (AL 15, AL 1849, H-2000-14, H-02-28) were found highly tolerant, five tolerant, nine moderately tolerant, four sensitive and six were found highly sensitive in water logging treatment. In case of salinity treatment, six genotypes were found tolerant, five moderately tolerant, three sensitive and 14 were found highly sensitive. Based on higher plant survival (\%) under both water logging and saline conditions, common genotypes were identified for their further use in breeding programme.

Keywords: Germination; abiotic stresses; phenotypic variability; stress tolerance.

\section{INTRODUCTION}

Pigeonpea (Cajanus cajan (L.) Millsp.) is an important legume crop widely grown in many parts of Indian sub-continent. It is mainly used as human food, animal feed and an effective green manure crop. It is adapted to a wide range of environments and cropping systems. Major abiotic stresses encountered by pigeonpea are water logging, salinity and drought. These abiotic stresses adversely affect its productivity causing severe yield losses $[1,2]$. Pigeonpea is reported to be highly sensitive to water logging $[3,4]$ and salinity $[5,6]$. Water logging and salinity stresses are important yield constraints in pigeonpea as water logging blocks oxygen supply to roots which hampers root permeability [7] and salinity impairs seed germination, reduces nodule formation, retards plant development and finally reduces crop yield [8]. It is reported that germination and early vegetative growth stages in pigeonpea are more affected by water logging than the flowering stage [9]. Water logging is reported to delay flowering, reduce vegetative growth, photosynthetic rate, biomass and grain yield $[10,11]$. It has also been reported that short duration varieties of pigeonpea are more prone to the risk of yield reduction due to water logging as compared to medium or long duration varieties [12]. Anoxic soil conditions are produced by excess water and consequently, plant roots suffer hypoxia or anoxia. Min and Bothalomew [13] reported gradual decrease in relative water content under water logging and plants wilt visually within a few hours of imposing water logging stress. It is observed that water logging induced decrease in leaf water potential [14] and membrane damage [15]. Water logging also caused reduction in stomata conductance and plants exposed to water logging exhibit increased stomata resistance and limited water uptake leading to internal water deficit [16].
Soil salinity is another major abiotic stress that affects plant growth, development and yield by causing physiological and biochemical changes in plants [17]. It leads to osmotic stress and interferes with mineral nutrients uptake [18]. Seedling and reproductive stages in plants are mainly affected by salinity stress [19]. Salinity together with water logging can cause deleterious effects in plants posing major threat to crop productivity [20]. These two abiotic stresses are related with each other as water logging results in rise of water table causing development of salinity in many parts of India [21]. In India, saline water are defined as water having $E C>2 \mathrm{dS} \mathrm{m}^{-1}[22,23]$. A large genetic variation has been found in different cultivated and wild species of pigeonpea for salinity tolerance [5,24]. In spite of the fact that both these stresses pose serious threat to pigeonpea production, very few efforts have been made to identify tolerant sources $[4,11]$. Thus, it becomes important to identify the diverse sources of pigeonpea tolerant to water logging and salinity stresses to develop high yielding and tolerant cultivars keeping in view the present climatic conditions [25]. Therefore, the present study was undertaken to screen and identify pigeonpea genotypes tolerant to water logging and salinity stresses under both in vitro and in vivo conditions.

\section{MATERIALS AND METHODS}

\subsection{Experimental Materials}

The experimental material comprised a set of 60 pigeonpea genotypes, including advance breeding lines and some commercially released cultivars, acquired from different sources namely, International Crops Research Institute for Semi-Arid Tropics (ICRISAT), Patancheru; 
CCS Haryana Agricultural University, Hisar; Indian Agricultural Research Institute, New Delhi and Punjab Agricultural University, Ludhiana.

\subsection{In vitro Screening}

The 60 genotypes were evaluated under in vitro conditions in the laboratory of Department of Plant Breeding and Genetics, Punjab Agricultural University, Ludhiana, India, using two treatments viz., water logging and salinity stresses during rainy seasons of 2012-13 and 2013-14. The water logging treatment comprised distilled water while the salinity treatment was having $30 \mathrm{mM}$ $\mathrm{NaCl}$ solution. Prior to the treatment, seeds of different genotypes were surface sterilized with $0.1 \%$ mercuric chloride for $1-2$ minutes and residual chlorine was eliminated by rinsing the seeds with sterile distilled water several times. Seeds were submerged in beaker containing 200 $\mathrm{ml}$ normal distilled water in case of water logging treatment and in $200 \mathrm{ml} 30 \mathrm{mM} \mathrm{NaCl}$ solution in case of salinity treatment, respectively, for 7 days $(168 \mathrm{hrs})$ at $25 \pm 1^{\circ} \mathrm{C}$. After the submergence treatment, seeds were then placed in sterilized petri-plates containing germination paper moistened with distilled water. For each genotype, 3 replications each were kept for both the treatments using 15 seeds per replication. Petri-plates were then kept in BOD incubator maintained at $25 \pm 1^{\circ} \mathrm{C}$ for 7 days.

\subsection{Observations}

Data were recorded after 7 days of incubation for both the treatments for germination percentage, seedling length (plumule and radicle), seedling dry weight (plumule and radicle) and seedling vigor index. Germination count was recorded by counting the number of germinated seeds out of the total seeds. Seed was considered to be germinated when emerging radicle elongated upto $1 \mathrm{~mm}$ length and then average germination percentage was computed. Seedling length was recorded by measuring the length of plumule and radicle and seedling dry weight was recorded after drying them in oven at $60^{\circ} \mathrm{C}$ for 3 days. Seedling vigor index was calculated using the formula: Seedling vigor index $(\mathrm{VI})=$ mean germination $\% \mathrm{X}$ mean seedling dry weight $(\mathrm{mg})$. Based on seedling vigor index, the genotypes were categorized as highly tolerant $(\mathrm{Vl}=>2000)$, tolerant $(\mathrm{Vl}=1501$ 2000), moderately tolerant ( $\mathrm{Vl}=1001-1500)$, sensitive (VI=501-1000) and highly sensitive $(\mathrm{Vl}=\leq 500)$.

\subsection{In vivo Screening}

Based on results of laboratory screening, a set of 28 genotypes, including highly tolerant to highly sensitive, was chosen for in vivo screening in the pots/field. Plants of 28 genotypes were raised in $27 \mathrm{~cm}$ diameter plastic pots containing $10 \mathrm{~kg}$ of soil fertilized with recommended fertilizers as per package of practices for pigeonpea. Seeds of each genotype were sown in pots and replicated thrice for both water logging and salinity treatments. After germination, six plants were maintained finally for recording observations. After 30 days of sowing, the pots were kept for 5 days in ponds of $20 \mathrm{ft} \times 10 \mathrm{ft}$ size containing normal water (water logging) and $30 \mathrm{mM} \mathrm{NaCl}$ solution (salinity). The water level was maintained at top surface level of pots. Five days after the water logging and salinity treatments, pots were removed from the ponds and placed on ground for the recovery of plants. During recovery of plants, normal water was applied as and when required.

\subsection{Observations}

Survival of plants was recorded after 5 days and then the final survival was recorded one month after water logging and salinity treatments. Chlorophyll content of plants on the third leaf from the top of plant was measured with SPAD (502-plus) before and 5 days after the water logging and salinity treatments. In each replication three plants were taken randomly for recording chlorophyll content. Percent reduction in chlorophyll content was calculated using the formula: Reduction in chlorophyll content $(\%)=$ (chlorophyll content before treatment chlorophyll content after treatment)/chlorophyll content before treatment $X 100$. As suggested by Dua [26], the genotypes were categorized based on final plant survival percentage (PS), as highly tolerant (>90\% PS), tolerant $(75-90 \%$ PS), moderately tolerant (50-74\% PS), sensitive (25$49 \%$ PS) and highly sensitive (<25\% PS). Data on initiation and completion of leaf senescence were also recorded visually after water logging and salinity treatments.

\subsection{Experimental Design and Statistical Analysis}

Both the experiments (in vitro and in vivo) were conducted following Completely Randomized Design with three replications. The analysis of variance (ANOVA) was performed on single year data as well as on pooled data of two years using 
statistical analysis as per the procedure given by Snedecor and Cochran [27] and adapted by Cheema and Singh [28] using the following equation: $Y_{i j e}=\mu+T_{i}+\beta j+k e+\varepsilon i j e$; where the parameter $\mu$ is an overall mean, ti are the treatment effects, $\beta \mathrm{j}$ are the block effects, ke are the years' effect and $\varepsilon$ ije is the random error. All comparisons were made at $5 \%$ level of significance. Tukey test was performed to group the genotypes based on germination percentage, seedling vigor index for in vitro and on plant survival for in vivo studies. The correlation coefficients were also worked out among the various traits for in vitro and in vivo experiments following Al-Jibouri et al. [29].

\section{RESULTS}

\subsection{In vitro Screening}

Analysis of variance, performed on single year data as well on pooled data of two years, revealed significant differences among the genotypes for all the traits studied viz., germination percentage, seedling length, seedling dry weight and seedling vigor index under both water logging and saline conditions (data not shown). In case of water logging conditions, six genotypes viz., AL 1756, AL 1849, AH-06-7, H-2000-14, H-2003-14 and ICP 5028 were found as highly tolerant, 10 genotypes (AL 15, AL 1758, AH-06-9, AH-06-12, H-2001-25, H02-28, ICPL 332, ICPA 2039, ICPL 20128, JBP $110 \mathrm{~B})$ as tolerant, 24 as moderately tolerant, 12 as sensitive and eight as highly sensitive (Table 1). Under saline conditions, none of the genotypes was found to be highly tolerant, however, seven genotypes viz., AL 1849, AH06-7, H-2000-14, H-2001-25, H-2003-14, ICP5028 and JBP 110B were found as tolerant, 14 as moderately tolerant, 17 as sensitive and 22 as highly sensitive (Table 1). Data on germination percentage, seedling length, seedling dry weight and seedling vigor index of 28 genotypes including some highly tolerant to highly sensitive genotypes are presented in Tables 2 and 3 , respectively for water logging and saline conditions. It can be seen that genotype $\mathrm{AH}-06-7$ had the maximum seedling vigor index (2655.0) followed by $\mathrm{H}-2000-14$ (2636.3), AL 1756 (2099.8) and AL 1849 (2091.0) under water logging condition. The germination percentage of highly tolerant and tolerant genotypes was more than $75 \%$. Out of the 60 genotypes, 3 highly sensitive genotypes recorded zero values for germination percentage, seedling length, seedling dry weight and seedling vigor index (data not shown). Under saline conditions, the genotype $\mathrm{H}-2000-14$ had the maximum seedling vigor index (1841.9) followed by ICP 5028 (1706.3), AH-06-7 (1668.5) and $\mathrm{H}$ 2001-25 (1656.4). Germination of tolerant genotypes was more than $75 \%$. Of the total 22 highly sensitive genotypes, five genotypes, having $0-15 \%$ germination, showed zero values for seedling length, seedling dry weight and seedling vigor index. However, some genotypes like AL 1760, ICPL 99051 and UPAS 120 recorded comparatively higher germination percentage $(30-38.5 \%)$, but their seedling vigor index values (180.0-231.0) were very low due to low seedling dry weight as a result of less seedling growth after germination due to sensitivity to saline conditions.

Overall under in vitro condition, it was observed that mean values of all the genotypes for all the traits studied like germination percentage, seedling length, seedling dry weight and seedling vigor index were lower under saline conditions (63.1\%, $6.4 \mathrm{~cm}, 15.4 \mathrm{mg}, 1090.9$, respectively) as compared to water logging conditions $(72.8 \%$, $7.7 \mathrm{~cm}, 19.1 \mathrm{mg}, 1477.3$, respectively). Overall there were 23 genotypes which showed similar reactions, i.e. tolerant ( 2 genotypes), moderately tolerant (5 genotypes), sensitive (8 genotypes) and highly sensitive (8 genotypes), under both water logging and salinity conditions. Of the six highly tolerant genotypes under water logging conditions, five genotypes namely $\mathrm{AL} 1849, \mathrm{AH}-$ 06-7, $\mathrm{H}-2000-14, \mathrm{H}-2003-14$ and ICP 5028 showed tolerant reaction, whereas one genotype, AL 1756 showed moderately tolerant reaction to saline conditions. Of the 10 tolerant genotypes under water logging conditions, two genotypes (H-2001-25, JBP 110B) showed the same reaction, i.e. tolerant, while seven genotypes namely AL 15, AL 1758, AH-06-9, AH-06-12, H02-28, ICPA 2039 and ICPL 20128 changed their reaction to moderately tolerant, and one genotype, ICPL 332 changed its reaction to highly sensitive under saline conditions. Of the 24 genotypes that showed moderately tolerant reaction under water logging conditions, five genotypes namely AL 1779, AL 1839, AH-07-3, H-02-59 and SGBS 6 showed similar reaction (moderately tolerant), while the remaining 19 genotypes changed their reaction to either sensitive or highly sensitive under saline conditions. On the other hand, interestingly the genotype AH-09-9, which was found sensitive under water logging conditions, showed moderately tolerant reaction under saline conditions. 
Table 1. Grouping of pigeonpea genotypes based on seedling Vigor Index (VI) evaluated under water logging and saline conditions in vitro

\begin{tabular}{|c|c|c|}
\hline \multirow{2}{*}{$\begin{array}{l}\text { Categories } \\
\text { based on } \\
\text { seedling } \\
\text { Vigor Index } \\
\text { (VI) }\end{array}$} & \multicolumn{2}{|l|}{ Name of genotypes (Number of genotypes) } \\
\hline & Water logging & Salinity \\
\hline $\begin{array}{l}\text { Highly } \\
\text { tolerant } \\
(\mathrm{VI}=>2000)\end{array}$ & $\begin{array}{l}\text { AL 1756, AL 1849,AH-06-7, H-2000-14, H- } \\
\text { 2003-14, ICP 5028 (Total=6) }\end{array}$ & - \\
\hline \multirow{2}{*}{$\begin{array}{l}\text { Tolerant } \\
(\mathrm{Vl}=1501- \\
2000)\end{array}$} & \multicolumn{2}{|c|}{ Common genotypes: $\mathrm{H}-2001-25, \mathrm{JBP} 110 \mathrm{~B}($ Total=2) } \\
\hline & $\begin{array}{l}\text { AL 15, AL 1758, AH-06-9, AH-06-12, } \\
\text { H-02-28, ICPL 332, ICPA 2039, } \\
\text { ICPL 20128(Total:2+8=10) }\end{array}$ & $\begin{array}{l}\text { AL 1849, AH-06-7, H-2000-14, } \\
\text { H-2003-14, ICP } 5028 \\
\text { (Total: } 2+5=7 \text { ) }\end{array}$ \\
\hline \multirow{2}{*}{$\begin{array}{l}\text { Moderately } \\
\text { Tolerant } \\
(\mathrm{VI}=1001- \\
1500)\end{array}$} & \multicolumn{2}{|c|}{ Common genotypes: AL 1779, AL 1839, AH-07-3, H-02-59, SGBS 6 (Total=5) } \\
\hline & $\begin{array}{l}\text { AL 1812, AL 1847, AL 1873, AH-06-5, H-03- } \\
29, \mathrm{H}-03-30, \mathrm{H}-2005-5 \text {, Pusa 992, Pusa } \\
\text { 2002, Pusa 2001-10, Pusa 2010-5-2, Pusa } \\
\text { 2012-2, ICP 8857, ICP 14085, } \\
\text { ICPL 2376, LRG 30, RG-06-1, ASJ 123, Sel. } \\
\text { 107-1 (Total:5+19=24) }\end{array}$ & $\begin{array}{l}\text { AL 15, AL 1756, AL 1758, } \\
\text { AH-06-9, AH-09-9, AH-06-12, } \\
\text { H-02-28, ICPA 2039, ICPL } 20128 \\
\text { (Total:5+9=14) }\end{array}$ \\
\hline \multirow{2}{*}{$\begin{array}{l}\text { Sensitive } \\
(\mathrm{Vl}=501- \\
1000)\end{array}$} & \multicolumn{2}{|c|}{$\begin{array}{l}\text { Common genotypes: AL 201, AL 1747, AL 1881, PAU 881, AH-06-1, } \\
\text { Pusa 2011-1, ICP 4924,ICPL } 99050 \text { (Total=8) }\end{array}$} \\
\hline & $\begin{array}{l}\text { AL 1760, AH-09-9, ICPL 96061, } \\
\text { ICPL 99051 (Total:8+4=12) }\end{array}$ & $\begin{array}{l}\text { AL 1812, AH-06-5, H-03-29, } \\
\text { H-2005-5, Pusa 992, Pusa 2001- } \\
\text { 10, ICP 8857, ICP 14085, LRG } 30 \\
\text { (Total: } 8+9=17 \text { ) }\end{array}$ \\
\hline \multirow{2}{*}{$\begin{array}{l}\text { Highly } \\
\text { Sensitive } \\
(\mathrm{VI}=<500)\end{array}$} & \multicolumn{2}{|c|}{$\begin{array}{l}\text { Common genotypes: AL 1593, H-61-21, Pusa 33, MAL 9, MAL 12, MAL 15,ICP } \\
\text { 11811,UPAS } 120 \text { (Total=8) }\end{array}$} \\
\hline & - & $\begin{array}{l}\text { AL 1760, AL 1847, AL 1873, } \\
\text { H-03-30, Pusa 2002, Pusa 2010-5- } \\
\text { 2, Pusa 2012-2, ICPL 332, ICPL } \\
\text { 2376, ICPL 99051, ICPL 96061, } \\
\text { RG-06-1, ASJ 123, Sel. 107-1 } \\
\text { (Total: 8+14=22) }\end{array}$ \\
\hline
\end{tabular}

Correlation coefficients between various traits, worked out for the 28 genotypes studied under in vitro conditions, are presented in Table 4 . The results showed highly significant positive correlation among germination percentage, seedling length, seedling dry weight and seedling vigor index under both water logging and saline conditions.

\subsection{In vivo Screening}

A set of 28 genotypes was also evaluated under field (in vivo) conditions for chlorophyll content and plant survival (\%) under water logging and salinity conditions. Analysis of variance, performed on single year data as well on pooled data of two years, revealed significant differences for chlorophyll content before as well as after treatments, reduction in chlorophyll content after treatment and for plant survival percentage under both water logging and saline conditions (data not shown). It was observed that four genotypes namely AL 1849, H-02-28, AL 15 and $\mathrm{H}-2000-14$ showed $100 \%$ plant survival and very less reduction $(<10.0 \%)$ in chlorophyll content, and thus, categorized as highly tolerant, whereas five genotypes namely $\mathrm{AH}-06-12, \mathrm{H}$ 2003-14, AL 1756, AH-06-9 and AL 1758 showed comparatively less plant survival $(75.0$ to $83.0 \%$ ) and higher reduction (11.76 to $20.24 \%$ ) in chlorophyll content, and thus, categorized as tolerant under water logging conditions (Table 5). Of the remaining 19 genotypes, nine genotypes were categorized as moderately tolerant, four as sensitive and six genotypes as highly sensitive based on final plant survival percentage. The reduction in chlorophyll content was found to be $<10 \%$ in highly tolerant genotypes, while it was $>31 \%$ in sensitive or highly sensitive genotypes. 
Table 2. Germination percentage, seedling length, dry seedling weight, seedling vigor index and tolerance category of pigeonpea genotypes under water logging conditions (mean of two years' data)

\begin{tabular}{|c|c|c|c|c|c|c|}
\hline Sr. no. & Genotypes & $\begin{array}{l}\text { Germination } \\
(\%)\end{array}$ & $\begin{array}{l}\text { Seedling } \\
\text { length } \\
(\mathrm{mm})\end{array}$ & $\begin{array}{l}\text { Dry seedling } \\
\text { weight }(\mathrm{mg})\end{array}$ & $\begin{array}{l}\text { Seedling } \\
\text { Vigor Index } \\
\text { (VI) }\end{array}$ & $\begin{array}{l}\text { Tolerance } \\
\text { category* }\end{array}$ \\
\hline 1 & AH-06-7 & $90.0^{\text {a\# }}$ & 10.6 & 29.5 & $2655.0^{\mathrm{a}}$ & HT \\
\hline 2 & $\mathrm{H}-2000-14$ & $92.5^{\mathrm{a}}$ & 10.5 & 28.5 & $2636.3^{\mathrm{a}}$ & HT \\
\hline 3 & AL 1756 & $92.5^{\mathrm{a}}$ & 7.7 & 22.7 & $2099.8^{\mathrm{ab}}$ & HT \\
\hline 4 & AL 1849 & $82.0^{\mathrm{a}}$ & 11.9 & 25.5 & $2091.0^{\mathrm{abc}}$ & $\mathrm{HT}$ \\
\hline 5 & $\mathrm{H}-2003-14$ & $90.0^{\mathrm{a}}$ & 8.5 & 22.5 & $2025.0^{\mathrm{abc}}$ & HT \\
\hline 6 & ICP 5028 & $87.5^{\mathrm{a}}$ & 9.4 & 23.0 & $2012.5^{\mathrm{abc}}$ & HT \\
\hline 7 & $\mathrm{H}-2001-25$ & $81.5^{\mathrm{a}}$ & 8.9 & 23.5 & $1915.3^{b c}$ & $\mathrm{~T}$ \\
\hline 8 & AL 1758 & $79.5^{a}$ & 6.5 & 24.0 & $1908.0^{\mathrm{bc}}$ & $\mathrm{T}$ \\
\hline 9 & ICPA 2039 & $84.5^{\mathrm{a}}$ & 8.7 & 22.3 & $1884.3^{b c}$ & $\mathrm{~T}$ \\
\hline 10 & $\mathrm{H}-02-28$ & $85.0^{\mathrm{a}}$ & 8.8 & 22.1 & $1878.5^{\mathrm{bc}}$ & $\mathrm{T}$ \\
\hline 11 & $\mathrm{AH}-06-9$ & $75.0^{\mathrm{a}}$ & 9.0 & 24.7 & $1852.5^{\mathrm{bc}}$ & $\mathrm{T}$ \\
\hline 12 & ICPL 332 & $84.0^{\mathrm{a}}$ & 10.6 & 19.2 & $1612.8^{\mathrm{bcd}}$ & $\mathrm{T}$ \\
\hline 13 & ICPL 20128 & $83.0^{a}$ & 8.0 & 18.8 & $1560.4^{\text {bcde }}$ & $\mathrm{T}$ \\
\hline 14 & JBP 110B & $85.0^{\mathrm{a}}$ & 10.4 & 18.3 & $1555.5^{\text {bcde }}$ & $\mathrm{T}$ \\
\hline 15 & $\mathrm{AH}-06-12$ & $75.0^{\mathrm{a}}$ & 7.3 & 20.3 & $1512.4^{\text {bcdet }}$ & $\mathrm{T}$ \\
\hline 16 & $\mathrm{AL} 15$ & $75.0^{\mathrm{a}}$ & 7.0 & 20.0 & $1500.0^{\text {bcdet }}$ & $\mathrm{T}$ \\
\hline 17 & SGBS 6 & $72.5^{\mathrm{a}}$ & 7.4 & 20.0 & $1450.0^{\text {cdet }}$ & MT \\
\hline 18 & ICP 14085 & $70.0^{a}$ & 7.6 & 17.1 & $1197.0^{\operatorname{detg}}$ & MT \\
\hline 19 & $\mathrm{H}-02-59$ & $60.0^{\mathrm{a}}$ & 9.2 & 19.5 & $1170.0^{\operatorname{detg}}$ & MT \\
\hline 20 & Pusa 992 & $65.0^{\mathrm{a}}$ & 7.8 & 17.6 & $1144.0^{\operatorname{detg}}$ & MT \\
\hline 21 & $\mathrm{AH}-06-5$ & $67.5^{\mathrm{a}}$ & 8.1 & 16.6 & $1120.5^{\operatorname{detg}}$ & MT \\
\hline 22 & PAU 881 & $59.5^{\mathrm{a}}$ & 8.2 & 16.1 & $958.0^{\operatorname{detgh}}$ & $S$ \\
\hline 23 & AH-09-9 & $57.5^{\mathrm{ab}}$ & 6.4 & 16.2 & $931.5^{\operatorname{etgn}}$ & $S$ \\
\hline 24 & $\mathrm{AL} 1760$ & $61.0^{\mathrm{a}}$ & 5.6 & 14.2 & $866.2^{\text {tgh }}$ & $\mathrm{S}$ \\
\hline 25 & ICPL 99050 & $67.0^{\mathrm{a}}$ & 3.7 & 11.7 & $783.9^{\text {gn }}$ & $\mathrm{S}$ \\
\hline 26 & ICPL 99051 & $60.0^{\mathrm{a}}$ & 2.9 & 11.9 & $714.0^{\text {gn }}$ & $\mathrm{S}$ \\
\hline 27 & UPAS 120 & $45.0^{\mathrm{ab}}$ & 4.1 & 6.8 & $306.0^{\text {hi }}$ & $\mathrm{HS}$ \\
\hline \multirow[t]{4}{*}{28} & MAL 15 & $10.0^{\mathrm{b}}$ & 0.5 & 2.3 & $23.0^{\prime}$ & HS \\
\hline & Mean & 72.8 & 7.7 & 19.1 & 1477.3 & \\
\hline & $\mathrm{CD}(0.05)$ & 24.47 & 3.63 & 5.67 & 345.34 & \\
\hline & MSD & 48.19 & & & 654.92 & \\
\hline
\end{tabular}

${ }^{*} H T$ : Highly tolerant (VI: >2000); T: Tolerant (VI: 1500-2000); MT: Moderately Tolerant (VI: 1000-1499.99); S: Sensitive (VI: 500-999.99; HS: Highly Sensitive (VI: <500); MSD: Minimum significant difference; \#Same letters indicate non-significant while different letters indicate significant differences among the genotypes

In case of saline conditions, none of the genotypes showed more than $90 \%$ plant survival, however six genotypes namely $\mathrm{AL} 1756, \mathrm{AL}$ 1849, H-2-28, H-2001-25, AL 1758 and $\mathrm{AL} 15$ showed higher plant survival (75-83\%) and less reduction $(<21.0 \%)$ in chlorophyll content, and thus, categorized as tolerant, whereas five genotypes namely PAU 881, H-2000-14, H-02259, SGBS 6 and $\mathrm{H}-2003-14$ showed moderate plant survival ranging from 56.0 to $66.0 \%$ and higher reduction ( 23.77 to $35.88 \%$ ) in chlorophyll content, and thus, categorized as moderately tolerant under saline conditions (Table 6). Of the remaining 17 genotypes, three genotypes were categorized as sensitive and 14 as highly sensitive based on final plant survival. The reduction in chlorophyll content was also found to be $<20 \%$ in tolerant genotypes, while it was $>37 \%$ in sensitive or highly sensitive genotypes.

Of the four genotypes which were found highly tolerant under water logging condition, three genotypes, $A L$ 1849, $\mathrm{H}-02-28$ and $A L$ 15, changed their category to tolerant, while one genotype, $\mathrm{H}-2000-14$ changed its category to moderately tolerant under saline conditions. Of the five tolerant genotypes under water logging condition, two genotypes ( $\mathrm{AL}$ 1756, $\mathrm{AL}$ 1758) gave similar reaction, while one genotype $(\mathrm{H}$ 2003-14) changed its reaction to moderately tolerant, and two genotypes (H-06-9, H-06-12) changed their reaction to highly sensitive 
under saline conditions. Of the nine moderately tolerant genotypes under water logging conditions, three genotypes (H-02-59, PAU 881, SGBS 6) gave similar reaction, while one genotype (AL 1760) changed its reaction to sensitive, four genotypes (ICPL 332, AH-09-9, $\mathrm{AH}-06-5, \mathrm{AH}-06-7)$ changed their reaction to highly sensitive, and interestingly one genotype, $\mathrm{H}-2001-25$, changed its reaction to tolerant under saline conditions.

Table 3. Germination percentage, seedling length, dry seedling weight, seedling vigor index and tolerance category of pigeonpea genotypes under saline $(30 \mathrm{mM} \mathrm{NaCl})$ conditions (mean of two years' data)

\begin{tabular}{|c|c|c|c|c|c|c|}
\hline Sr. no. & Genotypes & $\begin{array}{l}\text { Germination } \\
(\%)\end{array}$ & $\begin{array}{l}\text { Seedling } \\
\text { length }(\mathrm{mm})\end{array}$ & $\begin{array}{l}\text { Dry } \\
\text { seedling } \\
\text { weight } \\
\text { (mg) }\end{array}$ & $\begin{array}{l}\text { Seedling } \\
\text { Vigor Index } \\
\text { (VI) }\end{array}$ & $\begin{array}{l}\text { Tolerance } \\
\text { category }^{*}\end{array}$ \\
\hline 1 & $\mathrm{H}-2000-14$ & $81.5^{\text {a\# }}$ & 10.8 & 22.6 & $1841.9^{a}$ & $T$ \\
\hline 2 & ICP 5028 & $75.5^{\mathrm{ab}}$ & 7.0 & 22.6 & $1706.3^{\mathrm{ab}}$ & $T$ \\
\hline 3 & $\mathrm{AH}-06-7$ & $75.5^{\mathrm{ab}}$ & 9.6 & 22.1 & $1668.5^{\mathrm{ab}}$ & $\mathrm{T}$ \\
\hline 4 & H-2001-25 & $82.0^{\mathrm{a}}$ & 9.2 & 20.2 & $1656.4^{\mathrm{abc}}$ & $\mathrm{T}$ \\
\hline 5 & $\mathrm{H}-2003-14$ & $83.0^{\mathrm{a}}$ & 9.2 & 19.9 & $1651.7^{\mathrm{abc}}$ & $\mathrm{T}$ \\
\hline 6 & AL 1849 & $85.0^{a}$ & 8.1 & 19.4 & $1649.0^{\mathrm{abc}}$ & $\mathrm{T}$ \\
\hline 7 & JBP 110B & $82.5^{\mathrm{a}}$ & 7.4 & 18.3 & $1509.8^{\mathrm{abcd}}$ & $\mathrm{T}$ \\
\hline 8 & AL 1756 & $78.5^{\mathrm{ab}}$ & 5.7 & 18.4 & $1444.4^{\mathrm{abcd}}$ & MT \\
\hline 9 & ICPL 20128 & $78.5^{\mathrm{ab}}$ & 6.6 & 17.9 & $1405.1^{\text {abcde }}$ & MT \\
\hline 10 & SGBS 6 & $66.5^{\mathrm{abc}}$ & 8.4 & 21.9 & $1456.4^{\mathrm{abcd}}$ & MT \\
\hline 11 & $\mathrm{H}-02-28$ & $73.5^{\mathrm{ab}}$ & 7.1 & 18.4 & $1352.4^{\text {abcde }}$ & MT \\
\hline 12 & AL 1758 & $76.0^{\text {ab }}$ & 4.4 & 17.6 & $1337.6^{\text {abcde }}$ & MT \\
\hline 13 & $\mathrm{AL} 15$ & $72.5^{\mathrm{ab}}$ & 8.7 & 18.3 & $1326.8^{\text {abcde }}$ & MT \\
\hline 14 & ICPA 2039 & $75.0^{\text {ab }}$ & 6.7 & 16.2 & $1215.0^{\text {bcdet }}$ & MT \\
\hline 15 & AH-06-9 & $69.0^{\mathrm{abc}}$ & 6.2 & 16.6 & $1145.4^{\text {bcdet }}$ & MT \\
\hline 16 & $\mathrm{AH}-06-12$ & $58.5^{\mathrm{abc}}$ & 7.2 & 19.5 & $1140.8^{\text {bcdet }}$ & MT \\
\hline 17 & $\mathrm{H}-02-59$ & $69.0^{\mathrm{abc}}$ & 8.0 & 16.4 & $1131.6^{\text {bcdet }}$ & MT \\
\hline 18 & $\mathrm{AH}-09-9$ & $60.0^{\mathrm{abc}}$ & 7.0 & 17.3 & $1038.0^{\text {cdet }}$ & MT \\
\hline 19 & $\mathrm{AH}-06-5$ & $68.5^{a b c}$ & 6.7 & 14.5 & $993.3^{\text {det }}$ & S \\
\hline 20 & Pusa 992 & $60.0^{\mathrm{abc}}$ & 6.7 & 15.1 & $906.0^{\text {det }}$ & $\mathrm{S}$ \\
\hline 21 & PAU 881 & $66.5^{a b c}$ & 7.3 & 13.6 & $904.4^{\text {det }}$ & $\mathrm{S}$ \\
\hline 22 & ICP 14085 & $53.0^{a b c}$ & 6.0 & 15.2 & $805.6^{\text {etg }}$ & $\mathrm{S}$ \\
\hline 23 & ICPL 99050 & $60.0^{\mathrm{abc}}$ & 3.9 & 11.0 & $660.0^{\text {tg }}$ & $\mathrm{S}$ \\
\hline 24 & AL 1760 & $38.5^{\mathrm{bcd}}$ & 3.8 & 6.0 & $231.0^{\mathrm{gh}}$ & HS \\
\hline 25 & ICPL 99051 & $38.5^{\mathrm{bcd}}$ & 2.8 & 4.9 & $188.7^{\mathrm{gh}}$ & HS \\
\hline 26 & UPAS 120 & $30.0^{\mathrm{cd}}$ & 5.0 & 6.0 & $180.0^{\mathrm{gh}}$ & HS \\
\hline 27 & ICPL 332 & $5.0^{\mathrm{d}}$ & 0.0 & 0.0 & $0.0^{\mathrm{n}}$ & HS \\
\hline \multirow{4}{*}{28} & MAL 15 & $5.0^{d}$ & 0.0 & 0.0 & $0.0^{\mathrm{h}}$ & HS \\
\hline & Mean & 63.1 & 6.4 & 15.4 & 1090.9 & \\
\hline & CD (0.05) & 21.72 & 3.46 & 5.17 & 315.42 & \\
\hline & MSD & 40.57 & & & 626.72 & \\
\hline
\end{tabular}

* T: Tolerant (VI: 1500-2000); MT: Moderately Tolerant (VI: 1000-1499.99); S: Sensitive (VI: 500-999.99; HS: Highly Sensitive (VI: <500); MSD: Minimum significant difference; \#Same letters indicate non-significant while different letters indicate significant differences among the genotypes

Table 4. Correlation between various traits in pigeonpea genotypes under in vitro conditions

\begin{tabular}{lllllll}
\hline Traits & \multicolumn{2}{c}{$\begin{array}{c}\text { Seedling length } \\
(\mathbf{c m})\end{array}$} & \multicolumn{2}{c}{$\begin{array}{c}\text { Seedling dry weight } \\
(\mathbf{m g})\end{array}$} & \multicolumn{2}{c}{$\begin{array}{c}\text { Seedling vigor } \\
\text { index }\end{array}$} \\
\cline { 2 - 7 } & WL\# & SAL\# & WL & SAL & WL & SAL \\
\hline Germination\% & $0.782^{* *}$ & $0.841^{* *}$ & $0.872^{* *}$ & $0.917^{* *}$ & $0.899^{\star *}$ & $0.930^{* *}$ \\
Seedling length $(\mathrm{cm})$ & & & $0.839^{\star *}$ & $0.893^{\star *}$ & $0.801^{* *}$ & $0.858^{* *}$ \\
Seedling dry weight $(\mathrm{mg})$ & & & & & $0.967^{\star *}$ & $0.964^{* *}$ \\
\hline
\end{tabular}

\# WL: Water logging conditions; SAL: Saline conditions; **Significant at $1 \%$ level 
Table 5. Chlorophyll content and plant survival of some tolerant and sensitive pigeonpea genotypes under water logging conditions (mean of two years' data)

\begin{tabular}{|c|c|c|c|c|c|c|}
\hline \multirow[t]{2}{*}{ Sr. no. } & \multirow[t]{2}{*}{ Genotype } & \multicolumn{3}{|c|}{ Chlorophyll content } & \multirow{2}{*}{$\begin{array}{l}\text { Plant } \\
\text { survival } \\
\text { (\%) }\end{array}$} & \multirow{2}{*}{$\begin{array}{l}\text { Tolerance } \\
\text { category* }\end{array}$} \\
\hline & & $\begin{array}{l}\text { Before } \\
\text { treatment }\end{array}$ & $\begin{array}{l}\text { After } \\
\text { treatment }\end{array}$ & $\begin{array}{l}\text { Reduction after } \\
\text { treatment (\%) }\end{array}$ & & \\
\hline 1 & $\mathrm{AL} 1849$ & 43.6 & 41.3 & 5.28 & $100^{\mathrm{a \#}}$ & HT \\
\hline 2 & $\mathrm{H}-02-28$ & 43.1 & 39.3 & 8.82 & $100^{\mathrm{a}}$ & HT \\
\hline 3 & AL 15 & 39.2 & 35.6 & 9.18 & $100^{\mathrm{a}}$ & $\mathrm{HT}$ \\
\hline 4 & $\mathrm{H}-2000-14$ & 38.7 & 34.9 & 9.82 & $100^{\mathrm{a}}$ & HT \\
\hline 5 & $\mathrm{AH}-06-12$ & 42.5 & 37.5 & 11.76 & $83^{\mathrm{ab}}$ & $\mathrm{T}$ \\
\hline 6 & $\mathrm{H}-2003-14$ & 35.0 & 29.4 & 16.00 & $83^{\mathrm{ab}}$ & $\mathrm{T}$ \\
\hline 7 & AL 1756 & 41.6 & 36.1 & 13.22 & $80^{\mathrm{ab}}$ & $\mathrm{T}$ \\
\hline 8 & AH-06-9 & 47.2 & 40.5 & 14.19 & $80^{\mathrm{ab}}$ & $\mathrm{T}$ \\
\hline 9 & AL 1758 & 42.0 & 33.8 & 19.52 & $75^{\mathrm{abc}}$ & $\mathrm{T}$ \\
\hline 10 & ICPL 332 & 32.5 & 25.5 & 21.53 & $67^{\mathrm{abcd}}$ & MT \\
\hline 11 & $\mathrm{H}-2001-25$ & 47.3 & 36.4 & 23.04 & $67^{\mathrm{abcd}}$ & MT \\
\hline 12 & $\mathrm{AH}-09-9$ & 47.6 & 35.4 & 25.63 & $67^{\mathrm{abcd}}$ & MT \\
\hline 13 & $\mathrm{AL} 1760$ & 42.1 & 30.7 & 27.08 & $67^{\mathrm{abc}}$ & MT \\
\hline 14 & $\mathrm{H}-02-59$ & 37.9 & 26.7 & 29.55 & $66^{\text {abcde }}$ & MT \\
\hline 15 & PAU 881 & 43.4 & 32.4 & 25.35 & $60^{\text {abcde }}$ & MT \\
\hline 16 & AH-06-5 & 41.4 & 30.8 & 25.60 & $60^{\mathrm{abcde}}$ & MT \\
\hline 17 & SGBS 6 & 34.4 & 24.8 & 27.90 & $55^{\text {bcdet }}$ & MT \\
\hline 18 & $\mathrm{AH}-06-7$ & 41.1 & 32.2 & 21.65 & $50^{\text {bcdet }}$ & MT \\
\hline 19 & ICPA 2039 & 35.7 & 20.6 & 42.30 & $34^{\text {cdetg }}$ & $\mathrm{s}$ \\
\hline 20 & Pusa 992 & 38.2 & 21.4 & 43.98 & $33^{\text {cdetg }}$ & $\mathrm{S}$ \\
\hline 21 & ICP 5028 & 35.6 & 24.5 & 31.18 & $25^{\operatorname{detg}}$ & $\mathrm{S}$ \\
\hline 22 & UPAS 120 & 46.0 & 30.2 & 34.35 & $25^{\text {detg }}$ & $S$ \\
\hline 23 & JBP 110B & 32.5 & 19.5 & 40.00 & $23^{\text {etg }}$ & $\mathrm{HS}$ \\
\hline 24 & ICPL 20128 & 36.7 & 21.4 & 41.69 & $15^{\text {tg }}$ & HS \\
\hline 25 & MAL 15 & 33.3 & 19.3 & 42.04 & $0^{9}$ & HS \\
\hline 26 & ICP 14085 & 30.2 & 16.5 & 45.36 & $0^{g}$ & HS \\
\hline 27 & ICPL 99051 & 38.4 & 20.5 & 46.61 & $0^{g}$ & HS \\
\hline \multirow{4}{*}{28} & ICPL 99050 & 36.2 & 18.6 & 48.62 & $0^{9}$ & HS \\
\hline & Mean & 39.41 & 29.15 & 26.78 & 54.11 & \\
\hline & CD (0.05) & 12.43 & 11.26 & 17.67 & 21.46 & \\
\hline & MSD & & & & 42.36 & \\
\hline
\end{tabular}

In water logging treatment, the leaf senescence started on $4^{\text {th }}$ day from the lower part of plants and moved upwards and was nearly $100 \%$ after $10^{\text {th }}$ day of treatment in highly sensitive genotypes such as MAL 15, ICP 14085, ICPL99051 and ICPL 99050. In case of salinity treatment, the leaf senescence started on $2^{\text {nd }}$ day of the treatment in similar fashion as in case of water logging treatment and was nearly $100 \%$ after $6^{\text {th }}$ day of treatment in highly sensitive genotypes such as ICPL 14085, ICPL 99051, MAL 15, ICP 5028, ICPL 332, AH-06-9, AH-06-9 and ICPL 99050. In rest of the highly sensitive genotypes, complete leaf senescence was observed after 15-20 days of treatment. Comparatively lesser leaf senescence was observed in highly tolerant genotypes, while low to moderate leaf senescence was recorded in tolerant or moderately tolerant genotypes under both water logging and saline conditions.

Chlorophyll content measured before the water logging and salinity treatments revealed that the genotype $\mathrm{AH}-09-9$ had the maximum value (47.6) followed by H-2001-25 (47.3) and $\mathrm{AH}-06-9$ (47.2). After water logging treatment, the maximum chlorophyll content was observed in AL 1849 (41.3) followed by AH-06-9 (40.5) and $\mathrm{H}-02-28$ (39.3), whereas after salinity treatment, the maximum chlorophyll content was recorded by $\mathrm{H}-2001-25$ (38.5) followed by AL 1849 (37.6) and AL 1756 (37.3). Overall, there were nine genotypes (highly tolerant or tolerant) under water logging conditions and six genotypes 
(tolerant) under saline conditions that showed $<20 \%$ reduction in chlorophyll content (Tables 5 , 6 ). On the average of all genotypes under in vivo conditions, it was observed that reduction in chlorophyll content was higher (36.19\%) in saline conditions compared to water logging conditions (26.78\%).

Results of the present study revealed that five genotypes namely AL 1756, AL 1849, H-2-28, AL 1758 and $\mathrm{AL} 15$ recorded higher plant survival $(>75 \%)$ under water logging as well as saline conditions, whereas six genotypes namely JBP 110B, ICPL 20128, MAL 15, ICP 14085, ICPL 99051 and ICPL 99050 were found highly sensitive under both conditions. The correlation coefficients between different traits, worked out for the 28 genotypes studied under in vivo conditions, are presented in Table 7. Plant survival percentage showed highly significant positive correlation with chlorophyll content both before and after water logging treatment. The plant survival also showed highly significant positive correlation with chlorophyll content after saline treatment, while before saline treatment the correlation between these two traits was nonsignificant. The reduction in chlorophyll content showed highly significant negative correlation with plant survival percentage and chlorophyll content both after the water logging and saline treatments. The reduction in chlorophyll content also showed significant negative correlation with chlorophyll content before water logging treatment, while after salinity treatment

Table 6. Chlorophyll content and plant survival of some tolerant and sensitive pigeonpea genotypes under saline conditions (mean of two years' data)

\begin{tabular}{|c|c|c|c|c|c|c|}
\hline \multirow[t]{2}{*}{ Sr. no. } & \multirow[t]{2}{*}{ Genotype } & \multicolumn{3}{|c|}{ Chlorophyll content } & \multirow{2}{*}{$\begin{array}{l}\text { Plant } \\
\text { survival } \\
\text { (\%) }\end{array}$} & \multirow{2}{*}{$\begin{array}{l}\text { Tolerance } \\
\text { category }^{*}\end{array}$} \\
\hline & & $\begin{array}{l}\text { Before } \\
\text { treatment }\end{array}$ & $\begin{array}{l}\text { After } \\
\text { treatment }\end{array}$ & $\begin{array}{l}\text { Reduction after } \\
\text { treatment (\%) }\end{array}$ & & \\
\hline 1 & $\mathrm{AL} 1756$ & 41.6 & 37.3 & 10.34 & $83^{\text {a\# }}$ & $\mathrm{T}$ \\
\hline 2 & AL 1849 & 43.6 & 37.6 & 13.76 & $83^{\mathrm{a}}$ & $\mathrm{T}$ \\
\hline 3 & $\mathrm{H}-02-28$ & 43.1 & 36.1 & 16.24 & $78^{\mathrm{ab}}$ & $\mathrm{T}$ \\
\hline 4 & $\mathrm{H}-2001-25$ & 47.3 & 38.5 & 18.60 & $75^{\mathrm{ab}}$ & $\mathrm{T}$ \\
\hline 5 & AL 1758 & 42.0 & 36.1 & 14.05 & $75^{\mathrm{ab}}$ & $\mathrm{T}$ \\
\hline 6 & AL 15 & 39.2 & 31.6 & 19.38 & $75^{\mathrm{ab}}$ & $\mathrm{T}$ \\
\hline 7 & PAU 881 & 43.4 & 30.5 & 29.72 & $66^{\mathrm{abc}}$ & MT \\
\hline 8 & $\mathrm{H}-2000-14$ & 38.7 & 29.5 & 23.77 & $62^{\mathrm{abc}}$ & MT \\
\hline 9 & H-02-59 & 37.9 & 24.3 & 35.88 & $62^{\mathrm{abc}}$ & MT \\
\hline 10 & SGBS 6 & 34.4 & 22.7 & 34.01 & $58^{\mathrm{abcd}}$ & MT \\
\hline 11 & $\mathrm{H}-2003-14$ & 35.0 & 24.3 & 30.57 & $56^{\text {abcde }}$ & MT \\
\hline 12 & AL 1760 & 42.1 & 26.4 & 37.29 & $44^{\text {bcdet }}$ & $S$ \\
\hline 13 & Pusa 992 & 38.2 & 20.2 & 47.12 & $37^{\text {cdetg }}$ & $S$ \\
\hline 14 & ICPA 2039 & 35.7 & 18.6 & 47.90 & $37^{\text {cdetg }}$ & $S$ \\
\hline 15 & UPAS 120 & 46.0 & 27.6 & 40.00 & $23^{\operatorname{detg}}$ & HS \\
\hline 16 & AH-06-5 & 41.4 & 25.4 & 38.65 & $20^{\text {etg }}$ & HS \\
\hline 17 & ICPL 20128 & 36.7 & 22.3 & 39.24 & $18^{\text {tg }}$ & HS \\
\hline 18 & JBP $110 \mathrm{~B}$ & 32.5 & 17.8 & 45.23 & $18^{\text {tg }}$ & HS \\
\hline 19 & $\mathrm{AH}-06-12$ & 42.5 & 24.3 & 42.82 & $11^{\text {tg }}$ & HS \\
\hline 20 & $\mathrm{AH}-06-7$ & 41.1 & 24.5 & 40.39 & $6^{g}$ & HS \\
\hline 21 & ICP 14085 & 30.2 & 17.6 & 41.72 & $0^{g}$ & HS \\
\hline 22 & ICPL 99051 & 38.4 & 21.3 & 44.53 & $0^{9}$ & HS \\
\hline 23 & MAL 15 & 33.3 & 17.8 & 46.55 & $0^{g}$ & HS \\
\hline 24 & ICP 5028 & 35.6 & 18.7 & 47.47 & $0^{g}$ & HS \\
\hline 25 & ICPL 332 & 32.5 & 16.7 & 48.62 & $0^{g}$ & HS \\
\hline 26 & AH-06-9 & 47.2 & 23.4 & 50.42 & $0^{9}$ & HS \\
\hline 27 & $\mathrm{AH}-09-9$ & 47.6 & 22.3 & 53.15 & $0^{g}$ & HS \\
\hline \multirow[t]{4}{*}{28} & ICPL 99050 & 36.2 & 16.3 & 54.97 & $0^{g}$ & HS \\
\hline & Mean & 39.40 & 25.33 & 36.19 & 35.25 & \\
\hline & $\mathrm{CD}(0.05)$ & 12.43 & 10.32 & 19.17 & 18.76 & \\
\hline & MSD & & & & 37.59 & \\
\hline
\end{tabular}

HS: Highly Sensitive (<25\%PS); MSD: Minimum significant difference; \#Same letters indicate non-significant while different letters indicate significant differences among the genotypes 
Table 7. Correlation between various traits in pigeonpea genotypes under in vivo conditions

\begin{tabular}{|c|c|c|c|c|c|c|}
\hline \multirow[t]{2}{*}{ Traits } & \multicolumn{2}{|c|}{$\begin{array}{c}\text { Chlorophyll content } \\
\text { before treatment }\end{array}$} & \multicolumn{2}{|c|}{$\begin{array}{c}\text { Chlorophyll content } \\
\text { after treatment }\end{array}$} & \multicolumn{2}{|c|}{$\begin{array}{c}\text { Reduction in } \\
\text { chlorophyll content (\%) }\end{array}$} \\
\hline & WL\# & SAL\# & WL & SAL & WL & SAL \\
\hline Plant s & $0.498^{\star *}$ & 0.286 & 0.87 & 0.8 & $-0.950^{* \star}$ & $-0.902^{* *}$ \\
\hline $\begin{array}{l}\text { Chlorophyll content } \\
\text { before treatment }\end{array}$ & & & $0.799^{* *}$ & $0.652^{* *}$ & $-0.481^{\star *}$ & -0.309 \\
\hline $\begin{array}{l}\text { Chlorophyll content } \\
\text { after treatment }\end{array}$ & & & & & $-0.908^{* *}$ & $-0.919^{* *}$ \\
\hline
\end{tabular}

\# WL: Water logging conditions; SAL: Saline conditions; * Significant at 1\% level

the correlation was non-significant between these two traits.

Considering results of both in vitro and in vivo conditions together, one genotype, AL 1849, was found highly tolerant or tolerant under both water logging and saline conditions, while six genotypes namely $\mathrm{H}-2000-14, \mathrm{H}-2003-14, \mathrm{H}-02-$ 28, AL 15, AL 1756 and AL 1758 were found highly tolerant or tolerant under water logging and tolerant or moderately tolerant under saline conditions. Four genotypes namely MAL 15, UPAS 120, ICPL 99050 and ICPL 99051 were found sensitive or highly sensitive to both water logging and saline treatments under in vitro and in vivo conditions.

\section{DISCUSSION}

The results of the present study indicated existence of large phenotypic variability among the genotypes screened in vitro under water logging and saline conditions. These results were in accordance with the results of previous studies [30-32]. None of the genotypes was found to be highly tolerant under saline conditions, while six genotypes were found highly tolerant under water logging conditions. However, some of the genotypes showed similar reactions under water logging and saline conditions, but majority of the genotypes changed their reaction and showed lower level of tolerance to saline conditions as compared to their reaction under water logging conditions. Also the overall lower mean values for all the traits like germination percentage, seedling length, seedling dry weight and seedling vigor index under saline conditions as compared to water logging conditions. These results indicated that most of the pigeonpea genotypes used in the present study were more sensitive to saline conditions as compared to water logging conditions. Water logging results in anoxic conditions due to which respiration and electron transport are inhibited and ATP formation is decreased resulting in low seed viability and poor germination $[33,34]$. Seed germination rate has been used as phenotypic marker for selection of salinity tolerant pigeonpea cultivars [35] where they observed genotypic variability to a greater extent under salt stress conditions. Earlier in chickpea, the parameters like germination percentage, radicle and plumule length and plant survival at maturity have been found effective in differentiating tolerant and sensitive genotypes under saline conditions [36].

The correlation coefficients between various traits indicated that genotypes having higher germination percentage will generally have higher seedling length, seedling dry weight and seedling vigor index. For instance the genotypes like AH-06-7 and H-2000-14 recorded higher germination percentage, also had higher seedling length, seedling dry weight and seedling vigor index, whereas genotypes like UPAS 120 and MAL 15 recorded lower germination percentage, also had lower seedling length, seedling dry weight and seedling vigor index. Seedling vigor index of seedlings was also found to be positively correlated with germination percentage, root length and seedling dry weight in previous studies $[37,38]$.

In case of in vivo studies, significant differences were observed for chlorophyll content before as well as after treatments, reduction in chlorophyll content after treatment and for plant survival under both water logging and saline conditions indicated the existence of large genetic variability for both water logging and salinity tolerance in pigeonpea. Reduction in survival rate might be due to anoxia caused by water logging and salinity stresses. Overall, the lower mean values for chlorophyll content after salinity treatment and plant survival and high values for reduction in chlorophyll content indicated that saline conditions adversely affected the metabolism of plants which led to plant mortality.

The results revealed large extent of phenotypic variability in pigeonpea germplasm for tolerance to water logging and salinity stresses based on 
plant survival and reduction in chlorophyll content under field conditions. This variability can be exploited in breeding programme for developing high yielding cultivars having tolerance to water logging and salinity stress conditions [39]. In chickpea also, plant survival was taken as an important parameter for identifying tolerant genotypes under salinity stress [26]. Generally, it has been observed that water logging leads to low levels of oxygen in plants leading to internal water deficit. Oxygen deficiency leads to decline in net photosynthetic rate [40] which is due to reduced chlorophyll content and leaf area [41] and also due to stomata closure under stress [42]. Water logging results in wilting, chlorosis, senescence and abscission of leaves which may be due to reduced uptake and mobilization of nutrients. Reduction in chlorophyll content was also reported in pigeonpea [43,44], wheat [45], maize [46] and Vigna sinensis [47]. The highly significant negative correlation between reduction in chlorophyll content and plant survival indicated that high reduction in chlorophyll after water logging and saline treatments disrupted plant metabolism which led to plant mortality.

The genotype AL 1849 which was found highly tolerant or tolerant in both in vitro and in vivo condition under both water logging and saline conditions and the six genotypes namely $\mathrm{H}$ 2000-14, H-2003-14, H-02-28, AL 15, AL 1756 and $A L 1758$ which were found highly tolerant or tolerant in water logging and tolerant or moderately tolerant under saline conditions can be further evaluated under field conditions for their utility as cultivar as such or as donor for breeding for water logging and salinity tolerance. The genotypes which were found highly tolerant or tolerant and highly sensitive in both in vitro and in vivo conditions under water logging and saline conditions can be used for developing appropriate populations for genetic studies. Subarao et al. [6] studied comparative salinity tolerance among pigeonpea genotypes and their wild relatives. A large genetic variation was also observed earlier in cultivated and wild species of pigeonpea, especially $C$. scarabaeoides, for salinity tolerance [5]. As the wild relatives of pigeonpea are rich reservoir of genes for various abiotic stresses, including water logging and salinity tolerance, they can be exploited for introgressing desirable genes into cultivated background to minimize the adverse effects caused by such stresses [48]. It will help to broaden the narrow genetic base of pigeonpea which has always been a limiting factor in its breeding $[37,49]$.

\section{CONCLUSIONS}

Results of the present study indicated that both water logging and saline conditions affect the germination under in vitro conditions and plant survival under in vivo conditions to varying extent suggesting that both stresses alone or together pose a serious threat to pigeonpea cultivation. Thus, a holistic approach towards the management of these stresses is needed by developing water logging and salinity tolerant cultivars. Some of the genotypes were found promising under both in vitro and in vivo conditions there by indicating correlation for seedling and adult plant tolerance which can be exploited in breeding programmes.

\section{COMPETING INTERESTS}

Authors have declared that no competing interests exist.

\section{REFERENCES}

1. Abdul W, Ishfaq AM, Ghulam Q, Ghulam M, Tariq M, Muhammad A. Effect of salinity on germination, growth, yield, ionic balance and solute composition of pigeonpea (Cajanus cajan (L.) Millsp). Pak. J. Bot. 2006;38(4):1103-1117.

2. Rodriguez M, Canales E, Hidalgo OB. Molecular aspects of abiotic stress in plants. Biotechlogia Aplicada. 2005;22: 1-10.

3. Chauhan YS, Silim SN, Kumar Rao JVDK, Johansen C. A pot technique to screen pigeonpea cultivars for resistance to water logging. J. Agron. Crop Sci. 1997;178:179183.

4. Perera AM, Pooni HS, Saxena KB. Components of genetic variation in short duration pigeonpea crosses under water logged conditions. J. Genet. Plant Breed. 2001;55:21-38.

5. Rao IM, Venkataratnam N, Sheldrake AR. Field screening of pigeonpea for tolerance to soil salinity. Int. Pigeonpea Newsl. 1981;1:23.

6. Subbarao GV, Johansen C, Jana MK, Rao JVDKK. Comparative salinity responses among pigeonpea genotypes and their wild relatives. Crop Sci. 1991;31:415-418.

7. Else MA, Davies WS, Malone M, Jackson MS. A negative hydraulic message from oxygen-deficient roots of tomato plant? Plant Physiol. 1995;109:1017-1024.

8. Greenway H, Munns R. Mechanisms of salt tolerance in non- halophytes. 
Ann. Rev. Plant Physiol. 1980;31:149190.

9. Singh K, Sharma SP, Singh TK, Singh Y. Effect of water logging on growth, yield and nutrient concentration of black gram and green gram under sub-tropical condition of Varanasi. Ann. Agri. Res. 1986;7:169-177.

10. Takele A, Mcdavid CR. The response of pigeonpea cultivars to short durations of water logging. Afr. Crop Sci. J. 1995;3:5158.

11. Sarode SB, Singh MN, Singh UP. Genetics of water logging tolerance in pigeonpea (Cajanus cajan (L.) Millsp). Indian J. Genet. PI. Breed. 2007;67:264-265.

12. Matsunaga R, Ito O, Tobita S, Rao TP. Response of the pigeonpea (Cajanus cajan (L.) Millsp.) to nitrogen application and temporary waterlogging. In: Kutschera $\mathrm{L}$, Hubl E, Lichtenegger E, Persson $\mathrm{H}$, Sobotic M, editors. IRR Symposium Wien University, Bodenkultur, Klagenfurt. 1991; 183-186.

13. Min XJ, Bothalomew DP. Effect of flooding and drought on ethylene metabolism, titratable acidity and fruiting of pineapple. Acta Hort. 2005;666:135-148.

14. Else MA, Coupland D, Dutton L, Jackson MB. Decreased root hydraulic conductivity reduces leaf water potential, initiates stomatal closure and slows leaf expansion in flooded plants of castor oil (Riccinus communis) despite diminished delivery of ABA from roots to shoots in xylem sap. Physiol. Plantarum. 2001;111:46-54.

15. Rawyler A, Arpagaus S, Braendle R. Impact of oxygen stress and energy availability on membrane stability of plant cells. Ann. Bot.-London. 2002;90:499-507.

16. Parent $\mathrm{C}$, Berger A, Folzer $\mathrm{H}$, Dat J, Crevocoeur M, Badot PM, Capelli N. A novel nonsymbiotic haemoglobin from oak: Cellular and tissue specificity of gene expression. New Phytol. 2008;177:142154.

17. Munns R. Comparative physiology of salt and water stress. Plant Cell Environ. 2002;25:239-250.

18. Chikelu M, Afza R, Jain SM, Gregorio GB, Zapata-Arias FJ. Induced mutations for enhancing salinity tolerance in rice. In: Jenks MA, Hasegawa PM, Jain SM, editors. Advances in Molecular Breeding toward Drought and Salt Tolerant Crops, Springer The Netherlands. 2007;413-454.

19. Munns R, James RA. Avenues for increasing salt tolerance of crops and role of physiologically based selection traits. Plant Soil. 2003;247:93-105.

20. Barrett-Lennard E. The interaction between water logging and salinity in higher plants; causes, consequences and implications. Plant and Soil. 2003;253: 35-54.

21. Choudhary AK, Sultana R, Pratap A, Nadarajan N, Jha UC. Breeding for abiotic stresses in pigeonpea. J. Food Leg. 2011a;24(3):165-174.

22. Minhas PS. Saline water management in India. Agr. Water Manage. 1996;30:1-24.

23. Choudhary OP, Grattan SR, Minhas PS. Sustainable crop production using saline and sodic irrigation waters. In: Lichtfouse $\mathrm{E}$, editor. Alternate Farming Systems, Biotechnology, Drought Stress and Ecological Fertilisation, Sustainable Agriculture Reviews 6. Springer Dordrecht, New York. 2011b;293-318.

24. Karajol K, Naik GR. 'Seed germination rate' as a phenotypical marker for selection of $\mathrm{NaCl}$ tolerant cultivars in pigeonpea (Cajanus cajan (L.) Millsp). World J. Sci. Technol. 2011;1(2):01-08.

25. Ceccarelli S, Grando S, Maatougui M, Michael M, Slash M, Haghparast R, et al. Plant breeding and climate changes. J. Agr. Sci.- Cambridge. 2010;148:627-637.

26. Snedecor GW, Cochran WC. Statistical methods. Eighth Edition, lowa State University Press; 1989.

27. Cheema HS, Singh B. Software statistical package CPCS-1. Department of Statistics, Punjab Agricultural University, Ludhiana, India; 1991.

28. Al-Jibouri HA, Miller PA, Robinson HF. Genetic and environmental variances and covariances in upland cotton cross of interspecific origin. Agron. J. 1958;50(10): 633-637.

29. Dubey SD, Asthana AN. Selection of plant type resistant to water logging in pigeonpea (abstract). In: Wallis ES, Byth $\mathrm{DE}$, editors. Food Legume Improvement for Asian Farming Systems. Proc. Int. Workshop held in Khon Kaen, Thailand 1-5 Sep, Canberra, Australia: ACIAR proceedings series. 1987;311:1986.

30. Krishnamurthy L, Upadhyaya HD, Saxena $\mathrm{KB}$, Vadez V. Variation for temporary water logging response within mini core pigeonpea germplasm. J. Agr. Sci.Cambridge. 2012;150:357-364.

31. Sultana R, Vales MI, Saxena KB, Rathore A, Rao S, Mula MG, Kumar RV. Water 
logging tolerance in pigeonpea (Cajanus cajan (L.) Millsp): genotypic variability and identification of tolerant genotypes. J. Agr. Sci.-Cambridge. 2012;151:659-671.

32. Johnson J, Cobb BG, Drew MC. Hypoxic induction of anoxia tolerance in root tips of Zea mays. Plant Physiol. 1989;91:837-841.

33. Tsai CF, Chu TM, Wang CY. Effect of water logging on growth and development of sorghum plant: responses of seed germination. Chinese Agron. J. 1997;7: 203-212.

34. Kumar RR, Krishna K, Naik GR. Variation of sensitivity to drought stress in pigeonpea (Cajanus cajan (L.) Millsp) cultivars during seed germination and early seedling growth. World J. Sci. Technol. 2011;1:11-18.

35. Chauhan R, Singh NP, Tiwari A, Singh A. Comparative genotypic tolerance against salinity in chickpea (Cicer arietinum L.) J. Food Leg. 2012;25(3):187-193.

36. Kumar J, Choudhary AK, Solanki R, Pratap A. Towards marker assisted selection in pulses - A review. Plant Breeding. 2011;130:297-313.

37. Bharathi M, Saxena KB. A comparative study of hybrid and inbred cultivars for germination and other related traits of pigeonpea. J. Food Leg. 2012;25(4):351354.

38. Dua RP, Sharma PC. Physiological basis of salinity tolerance in pigeonpea (Cajanus cajan (L.) and method of testing material in highly variable soil conditions. Indian J. Agric. Sci. 1996;66:405-412.

39. Dua RP. Differential response of chickpea (Cicer arietinum L.) genotypes to salinity. J. Agr. Sci.- Cambridge. 1992;119:367371.

40. Ashraf MA, Ahmad MSA, Ashraf M, Al Qurainy F, Ashraf MY. Alleviation of water logging stress in upland cotton (Gossypium hirsutum L.) by exogenous application of $\mathrm{K}^{+}$in soil and as a foliar spray. Crop Pasture Sci. 2011;62(1):25-38.

41. Malik AL, Colmer TD, Lamber H, Sehortemeyer M. Changes in physiological and morphological traits of roots and shoots of wheat in response to different depths of water logging. Aust. J. Plant Physiol. 2001;28:1121-1131.

42. Ashraf M, Arfan M. Gas exchange characteristics and water logging relations in two cultivars of Hibiscus esculentus under water logging. Biologia Plantarum. 2005;49:459-462.

43. Mumutha D, Ezhilmathi K, Sairam RK, Srivastava GC, Deshmukh PS, Meena RC. Water logging induced oxidative stress and anti-oxidant activity in pigeonpea genotypes. Biol. Plantarum. 2009;53(1):75-84.

44. Mumutha D, Sairam RK, Ezhilmathi K, Chinnusamy V, Meena RC. Effect of water logging on carbohydrate metabolism in pigeonpea (Cajanus cajan (L.): Upregualtion of sucrose synthase and alcohol dehydrogenase. Plant Sci. 2008; 175:706-716.

45. Collaku A, Harrison SA. Losses in wheat due to waterlogging. Crop Sci. 2002;42: 444-450.

46. Prasad S, Ram PC, Uma S. Effect of waterlogging duration on chlorophyll content, nitrate reductase activity, soluble sugars and grain yield of maize. Ann. Plant Physiol. 2004;18:1-5.

47. Younis ME, El-Shahaby OA, Nemat Alla MM, Bastawisy ZM. Kinetin alleviates the influence of waterlogging and salinity on growth and affects the production of plant growth regulators in Vigna sinensis and Zea mays. Agronomie. 2003;23:277-285.

48. Sharma HC, Pampapathy G, Reddy LJ. Wild relatives of pigeonpea as a source of resistance to the pod fly (Melanagromyza obtusa Malloch) and pod wasp (Tanaostigmodes cajaninae La Salle). Genet. Res. Crop Ev. 2003;50:817-824.

49. Yang SY, Pang W, Ash G, Harper J, Carling J, Wenzl P, Huttner E, Zong XX, Kilian A. Low level of genetic diversity in cultivated pigeonpea compared to its wild relatives is revealed by diversity arrays technology. Theor. Appl. Genet. 2006;113: 585-595.

(c) 2016 Singh et al.; This is an Open Access article distributed under the terms of the Creative Commons Attribution License (http://creativecommons.org/licenses/by/4.0), which permits unrestricted use, distribution, and reproduction in any medium, provided the original work is properly cited.

Peer-review history:

The peer review history for this paper can be accessed here: http://sciencedomain.org/review-history/14023 